\section{Mortality among Guarani Indians in Southeastern and Southern Brazil}

\author{
Mortalidade indígena Guarani no Sul e Sudeste \\ do Brasil
}

\author{
${ }_{1}$ Escola Nacional de Saúde \\ Pública Sergio Arouca, \\ Fundação Oswaldo Cruz, \\ Rio de Janeiro, Brasil. \\ 2 Museu Nacional, \\ Universidade Federal do Rio \\ de Janeiro, Rio de Janeiro, \\ Brasil. \\ Correspondence \\ A. M. Cardoso \\ Departamento de Endemias \\ Samuel Pessoa, Escola \\ Nacional de Saúde Pública \\ Sergio Arouca, Fundação \\ Oswaldo Cruz. \\ Rua Leopoldo Bulhões 1480 , \\ Rio de Janeiro, $R J$ \\ 21041-210, Brasil. \\ andrey@ensp.fiocruz.br
}

\begin{abstract}
Worldwide, indigenous peoples display a high burden of disease, expressed by profound health inequalities in comparison to non-indigenous populations. This study describes mortality patterns among the Guarani in Southern and Southeastern Brazil, with a focus on health inequalities. The Guarani population structure is indicative of high birth and death rates, low median age and low life expectancy at birth. The crude mortality rate (crude $M R=5.0 / 1,000$ ) was similar to the Brazilian national rate, but the under-five MR (44.5/1,000) and the infant mortality rate (29.6/1,000) were twice the corresponding MR in the South and Southeast of Brazil. The proportion of post-neonatal infant deaths was 83.3\%, 2.4 times higher than general population. The proportions of ill-defined (15.8\%) and preventable causes (51.6\%) were high. The principal causes of death were respiratory (40.6\%) and infectious and parasitic diseases (18.8\%), suggesting precarious living conditions and deficient health services. There is a need for greater investment in primary care and interventions in social determinants of health in order to reduce the health inequalities.
\end{abstract}

South American Indians; Respiratory Tract Infections; Mortality; Health Inequalities
Andrey Moreira Cardoso 1

Carlos E. A. Coimbra Jr. 1

Carla Tatiana Garcia Barreto 1

Guilherme Loureiro Werneck 1

Ricardo Ventura Santos 1,2

\section{Introduction}

Worldwide, indigenous peoples are among the social groups with the heaviest burden of disease 1,2 . The main characteristics of the morbidity and mortality patterns in indigenous peoples include high rates of infectious diseases, high infant and maternal mortality, and low life expectancy at birth 1 . The situation is also present in Latin America, where there are profound health inequalities between indigenous peoples and the non-indigenous population 3 .

Despite the wide range of studies on the epidemiology of mortality in the Brazilian population $4,5,6,7,8,9,10$, there are still few contributions that focus on indigenous mortality, especially considering the country's diversity of ethnic groups (over two hundred) 11,12,13,14,15,16. The relatively recent inclusion of the option indigena as a possible answer to the question on "race/color" in the data collection forms used in the national health information system 11 and national censuses 17 and the limited coverage and quality of the specific information system for indigenous peoples 18 create further difficulties and challenges for characterizing the mortality pattern of indigenous people in Brazil.

This article describes the mortality pattern of the Guarani indigenous people living in 83 villages in Southern and Southeastern Brazil, based on primary data from a surveillance system implemented for a case-control study on etiological 
factors for acute respiratory infections (ARI). The resulting mortality indicators are compared with other indigenous peoples and the general Brazilian population, with a focus on health inequalities related to ethnicity.

\section{Methods}

Data collection for this study took place from May 1st, 2007, to June 30th, 2008, covering 83 Guarani villages (M'Byá and NThandeva) located on the coast of the States of Rio de Janeiro (5), São Paulo (27), Paraná (4), and Santa Catarina (16), plus all the Guarani villages in the State of Rio Grande do Sul (32). These represent $98.8 \%$ of all the Guarani villages in the study area.

In 1999, Brazil established the nationwide Indigenous Health Care Sub-System (SIASI) under the Brazilian Unified National Health System (SUS), administered in the last ten years by the Brazilian National Health Foundation (Fundação Nacional de Saúde - FUNASA). The SIASI is organized in 34 Health Districts, which have districtlevel and local health coordinating bodies, where Multidisciplinary Indigenous Health Teams (EMSI) work. These teams are responsible for the primary health care in the villages, intended to be linked hierarchically and integrated with the other levels of complexity in the SUS 19.

The data were generated from a surveillance system implemented specifically for a case-control study on etiological factors for acute lower respiratory infections (ALRI) in indigenous children in the 83 villages, which included EMSI nurses trained for the study to perform surveillance in the field 20,21 .

We analyzed all the deaths that occurred during the period, with the respective underlying causes obtained from the death certificates, reported monthly to the study's surveillance system. The villages were visited every four months to perform an active search of unreported vital events. The procedures included checking the records of the EMSI teams and district and local health coordinating bodies, asking the nurses about the occurrence of deaths, and reviewing the hospital charts of children under five years hospitalized for ALRI, among other activities. Underlying causes of death were all coded by the same investigator (A.M.C.) according to the ICD1022 . Deaths from respiratory causes in children under five years were confirmed by interviewing the parents or guardians and reviewing the hospital charts.

The numerators for the mortality rates (MR) and the numerators and denominators for the proportional mortality indicators were com- posed from the deaths that occurred during the study period. The denominators for the MR were composed from the Guarani population living in the 83 villages at the middle of the study period (December 1st, 2007), according to data provided by the nurses in charge of the study surveillance system. To calculate the under-five child mortality rate, the denominator was the number of live births reported to the study surveillance system. The MR were initially calculated for the 14-month period and later transformed into annual rates.

A population pyramid was constructed. We also calculated male/female ratios by age bracket, population proportions by age bracket, and aging index. The latter also considered the criterion used by the Inter-Agency Health Information Network (Rede Interagencial de Informação para a Saúde-RIPSA) 23 , which defines elderly as $\geq 60$ years. Crude and age- and sex-standardized MR were also calculated by the direct method, using the Brazilian population according to the 2000 census and estimated for the year 200621 , in addition to specific MR by sex, age, and causes and proportional mortality by age and causes.

The Guarani mortality indicators were compared to the corresponding ones for the total Brazilian population and by regions of the country, using rates ratios and proportional ratios, with the following comparisons as the reference (1) indirect mortality estimates for under-five children for Brazil and its regions for the three-year period 1995-1997 24; (2) direct calculation and indirect estimates of mortality for Brazil and its regions for the year 2004 23; and (3) direct calculation of mortality based on data from the Mortality Information Systems (SIM) and Information System on Live Births (SINASC) for Brazil and by region for the years 2006 (Departamento de Informática do SUS. Informações de Saúde. http: / / www.datasus. gov.br, accessed on 17/Nov/2009).

\section{Ethics}

The research project was approved by the Southern Coastal District Indigenous Health Council (Conselho Distrital de Saúde Indígena do Litoral Sul), the Ethics Research Committee of the Brazilian National School of Public Health (Escola Nacional de Saúde Pública Sergio Arouca - ENSP), Oswaldo Cruz Foundation (Fundação Oswaldo Cruz - FIOCRUZ) (case review 130/05), and the National Commission on Ethics Research (Comissão Nacional de Ética em Pesquisa - CONEP, case review 154/2006). It was also authorized by the Brazilian National Indian Foundation (Fundação Nacional do Índio - FUNAI, 23/CGEP/07). 


\section{Results}

In December 2007, the total Guarani population in the 83 villages was 6,483 . During the study period, there were 347 live births and 38 deaths, of which 12 were under one year of age and 18 under five years of age.

The Guarani population pyramid has a broad base when compared to the general Brazilian population, narrowing rapidly with increasing age (Figure 1). In the Guarani, $20.2 \%, 52.1 \%$, and $4 \%$ of the individuals were under five years, under 15 years, and 65 years or older, respectively. The aging index was 7.7 individuals $\geq 65$ years for every 100 persons < 15 years, increasing to 9.8 for a cutoff of 60 years.

The male-female ratio was 108.8 males per 100 women. The male-female ratio at birth was 96.8 boys per 100 girls. There were more males up to 19 years of age $(0-4$ years: $118.5 ; 5$ - 19 years: 112.1). From 20 to 64 years of age, there were relatively more women (20-49 years: 99.3 ; 50-64 years: 86.0). From 65 years on, men predominated again ( $\geq 65$ years: 126.1 ).

The crude annual Guarani MR was 5.0/1,000 inhabitants. The specific female MR $(5.2 / 1,000)$ exceeded the male MR by $8.3 \%(4.8 / 1,000)\left(\mathrm{RR}_{\mathrm{f} / \mathrm{m}}\right.$ $=1.08$; 95\%CI: 0.55-2.17). Higher mortality rates occurred at the age extremes $(<1$ year: 29.6/1,000; and $\geq 50$ years: $20.1 / 1,000$ ), followed by $1-4$ years (14.8/1,000), 20-49 years $(2.7 / 1,000)$, and 5-19 years $(2.7 / 1,000)$.

The female MR exceeded the male rate in the $<1$ year bracket $\left(\mathrm{RR}_{\mathrm{f} / \mathrm{m}}=1.46\right.$; $95 \% \mathrm{CI}$ : 0.42-5.05) and 1-4 year bracket $\left(\mathrm{RR}_{\mathrm{f} / \mathrm{m}}=1.86\right.$; $95 \% \mathrm{CI}$ : 0.31 11.05). The female and male rates were similar after 50 years of age $\left(\mathrm{RR}_{\mathrm{f} / \mathrm{m}}=1.04 ; 95 \% \mathrm{CI}\right.$ : 0.31 3.56). The male MR exceeded the female rate in the 5-19-year bracket (when deaths occurred only in males), and in the 20-49-year bracket $\left(\mathrm{RR}_{\mathrm{f} / \mathrm{m}}\right.$ $=0.66$; 95\%CI: 0.11-3.95).

The overall standardized Guarani MR was lower than the corresponding MR estimated by RIPSA in all regions of Brazil in 2004. As for the rates calculated based on data from the Health Informatics Department (Departamento de Informática do SUS - DATASUS), the overall Guarani MR was similar to Brazil MR as a whole and the Northeast, higher than that of the North, and lower than the South and Southeast (Tables 1, 2 and 3 ). Standardized male mortality was higher in the general Brazilian population (Brazil: 6.5/1,000; Guarani: $4.4 / 1,000$ ), while in females the pattern was the opposite (Brazil: 4.5/1,000; Guarani: $6.0 / 1,000)$. The male Guarani MR reached $60-70 \%$ of the male rates in the general Brazilian

Figure 1

Comparison of the age and sex structures of the Guarani population in Southern and Southeastern Brazil, and of the general Brazilian population.

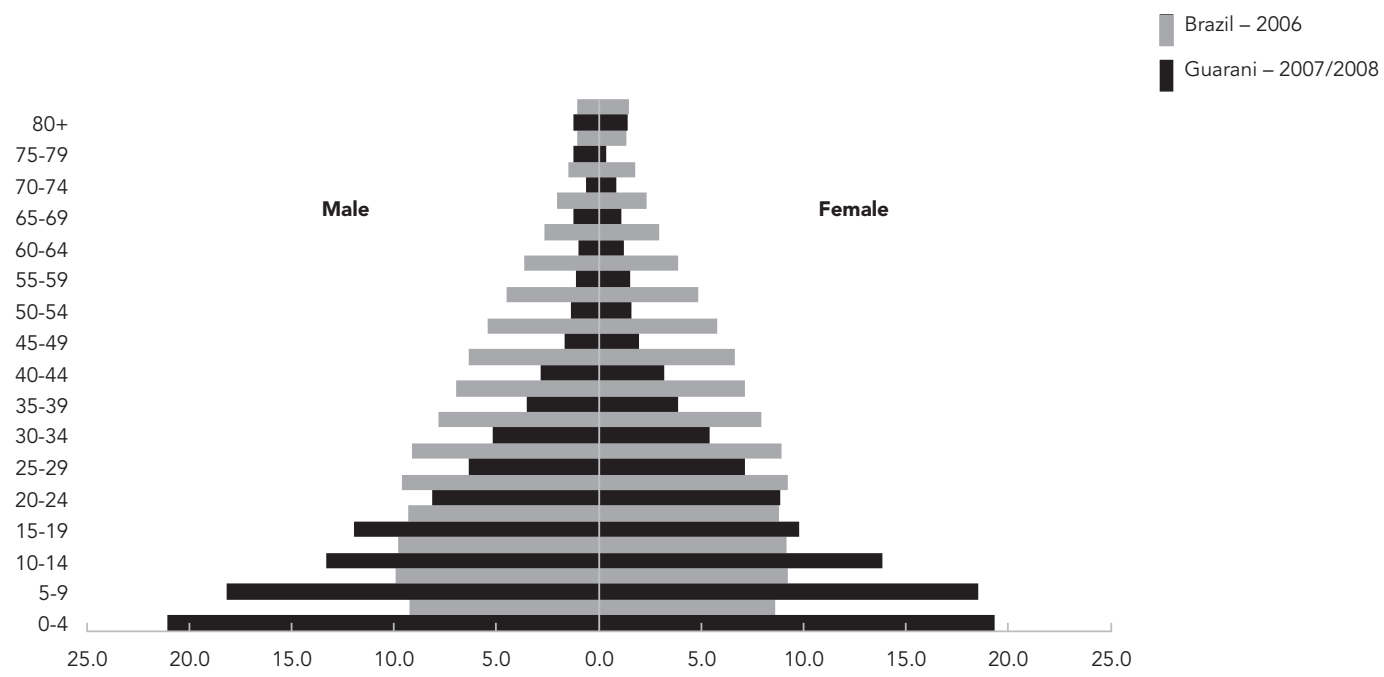

Source: Health Informatics Department (Departamento de Informática do SUS - DATASUS) [Cardoso] 20.

Note: population groups by age and sex presented as percentages of the total population of the respective sex. 
Guarani mortality indicators and comparisons with corresponding indicators for the non-indigenous population of Brazil and regions. Indirect estimates 1995-1997.

\begin{tabular}{|c|c|c|c|c|c|c|c|}
\hline \multirow[t]{2}{*}{ Indicators } & \multirow[t]{2}{*}{ Guarani } & \multicolumn{6}{|c|}{ Indirect estimates 1995-1997 * } \\
\hline & & Brazil ** & $N * *$ & NE ** & $\mathrm{CW} * *$ & $\mathrm{~S}$ ** & $\mathrm{SE} * \star$ \\
\hline \multirow{2}{*}{\multicolumn{8}{|c|}{$\begin{array}{l}\text { Overall and sex-specific MR } \\
(\text { per } 1,000) \star \star \star, \#\end{array}$}} \\
\hline & & & & & & & \\
\hline \multirow[t]{2}{*}{ Overall MR } & $5.3^{\star \star \star}$ & - & - & - & - & - & - \\
\hline & $5.0 \#$ & & & & & & \\
\hline Male MR & $4.4^{\star \star \star}$ & - & - & - & - & - & - \\
\hline Female MR & $6.0 * \star \star$ & - & - & - & - & - & - \\
\hline \multirow{2}{*}{\multicolumn{8}{|c|}{$\begin{array}{l}\text { Standardized specific MR by ICD-10 } \\
\text { chapters (per 100,000) \#\# }\end{array}$}} \\
\hline & & & & & & & \\
\hline MR, respiratory (Chap. X) & $124.3^{\star \star \star}$ & - & - & - & - & - & - \\
\hline MR, infectious/ parasitic (Chap. I) & $74.2 * \star \star$ & - & - & - & - & - & - \\
\hline MR, digestive system (Chap. XI) & 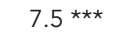 & - & - & - & - & - & - \\
\hline MR, circulatory system (Chap. IX) & $42.3^{\star \star \star}$ & - & - & - & - & - & - \\
\hline MR, external causes (Chap. XX) & $49.3^{\star \star \star}$ & - & - & - & - & - & - \\
\hline MR, other causes & 127.1 *** & - & - & - & - & - & - \\
\hline \multicolumn{8}{|l|}{ MR $<5$ yrs. and by specific causes (per } \\
\hline \multicolumn{8}{|l|}{1,000 live births) } \\
\hline $\mathrm{MR}<5$ yrs & 44.5 & - & - & - & - & - & - \\
\hline$M R<5$ yrs., ARI & 24.7 & - & - & - & - & - & - \\
\hline MR < 5 yrs., diarrhea & 7.4 & - & - & - & - & - & - \\
\hline MR $<5$ yrs., other causes & 12.4 & - & - & - & - & - & - \\
\hline Rates ratio ARI/diarrhea & 3.3 & - & - & - & - & - & - \\
\hline Rates ratio ARI/other causes & 2.0 & - & - & - & - & - & - \\
\hline \multicolumn{8}{|l|}{ IMR and components (per 1,000 } \\
\hline \multicolumn{8}{|l|}{ live births) } \\
\hline IMR & 29.6 & $37.5(0.8)$ & $36.2(0.8)$ & $60.5(0.5)$ & $25.8(1.1)$ & $22.8(1.3)$ & $25.9(1.1)$ \\
\hline Early neonatal IMR (0-6 days) & 4.9 & - & - & - & - & - & - \\
\hline Late neonatal IMR (7-27 days) & - & - & - & - & - & - & - \\
\hline Post-neonatal IMR (28-364 days) & 24.7 & - & - & - & - & - & - \\
\hline \multicolumn{8}{|l|}{ IMR by selected causes (per 1,000 live } \\
\hline \multicolumn{8}{|l|}{ births) } \\
\hline IMR from ARI & 17.3 & $3.8(4.6)$ & $3.2(5.4)$ & $5.9(2.9)$ & $2.3(7.5)$ & $2.5(6.9)$ & $2.8(6.2)$ \\
\hline IMR from diarrhea & 4.9 & $3.0(1.6)$ & $3.3(1.5)$ & $9.3(0.5)$ & $1.7(2.9)$ & $1.1(4.5)$ & $1.2(4.1)$ \\
\hline IMR from other causes & 7.4 & - & - & - & - & - & - \\
\hline Rates ratio ARI/diarrhea & 3.5 & - & - & - & - & - & - \\
\hline Rates ratio ARI/other causes & 2.3 & - & - & - & - & - & - \\
\hline \multicolumn{8}{|l|}{ Age-proportional mortality (\%) } \\
\hline$<5$ yrs. & 48.6 & $9.8(5.0)$ & $18.9(2.6)$ & $13.7(3.5)$ & $11.4(4.3)$ & $7.4(6.6)$ & $8.1(6.0)$ \\
\hline $1-4 \mathrm{yrs}$ & 16.2 & $9.0(1.8)$ & $3.2(5.1)$ & $2.1(7.7)$ & $1.9(8.5)$ & $1.1(14.7)$ & $1.1(14.7)$ \\
\hline$<1 \mathrm{yr}$ & 32.4 & $8.4(3.9)$ & $15.7(2.1)$ & $11.6(2.8)$ & $9.5(3.4)$ & $6.3(5.1)$ & $7.0(4.6)$ \\
\hline Early neonatal (0-6 days) & 16.7 & - & - & - & - & - & - \\
\hline Late neonatal (7-27 days) & - & - & - & - & - & - & - \\
\hline Post-neonatal (28-364 days) & 83.3 & $40.8(2.0)$ & $39.9(2.1)$ & $49.5(1.7)$ & $36.9(2.3)$ & $40.8(2.0)$ & $35.0(2.4)$ \\
\hline
\end{tabular}

(continues) 
Table 1 (continued)

\begin{tabular}{|c|c|c|c|c|c|c|c|}
\hline \multirow[t]{2}{*}{ Indicators } & \multirow[t]{2}{*}{ Guarani } & \multicolumn{6}{|c|}{ Indirect estimates $1995-1997$ * } \\
\hline & & Brazil ** & $\mathbf{N} * \star$ & NE ** & $\mathrm{CW} \star \star$ & $\mathrm{S} * \star$ & SE ** \\
\hline \multirow{2}{*}{\multicolumn{8}{|c|}{$\begin{array}{l}\text { Proportion of deaths from ill-defined } \\
\text { causes (\%) }\end{array}$}} \\
\hline & & & & & & & \\
\hline Overall population & 15.8 & - & - & - & - & - & - \\
\hline$<5$ yrs. & 22.2 & - & - & - & - & - & - \\
\hline $1-4$ yrs. & 33.3 & $18.6(1.8)$ & $27.1(1.2)$ & $31.5(1.1)$ & $8.3(4.0)$ & $8.1(4.1)$ & $10.7(3.1)$ \\
\hline$<1$ yr. & 16.7 & $12.6(1.3)$ & $16.0(1.0)$ & $25.5(0.7)$ & $6.0(2.8)$ & $6.3(2.7)$ & $5.0(3.3)$ \\
\hline \multicolumn{8}{|c|}{$\begin{array}{l}\text { Proportional mortality by groups of } \\
\text { defined causes (ICD-10) in } \\
\text { population (\%) }\end{array}$} \\
\hline Respiratory (Chap. X) & 40.6 & - & - & - & - & - & - \\
\hline Infectious/ parasitic (Chap. I) & 18.8 & - & - & - & - & - & - \\
\hline Digestive (Chap. XI) & 12.5 & - & - & - & - & - & - \\
\hline Circulatory (Chap. IX) & 9.4 & - & - & - & - & - & - \\
\hline External causes (Chap. XX) & 9.4 & - & - & - & - & - & - \\
\hline Other causes & 9.4 & - & - & - & - & - & - \\
\hline \multicolumn{8}{|c|}{$\begin{array}{l}\text { Proportional mortality by groups of } \\
\text { defined causes (ICD-10), < } 5 \text { yrs. (\%) }\end{array}$} \\
\hline ARI & 71.4 & - & - & - & - & - & - \\
\hline Diarrhea & 21.4 & - & - & - & - & - & - \\
\hline Other causes & 7.1 & - & - & - & - & - & - \\
\hline \multirow{2}{*}{\multicolumn{8}{|c|}{$\begin{array}{l}\text { Proportional mortality by groups of } \\
\text { defined causes (ICD-10), 1-4 yrs. (\%) }\end{array}$}} \\
\hline & & & & & & & \\
\hline ARI & 75.0 & $23.8(3.2)$ & $23.8(3.2)$ & $27(2.8)$ & $17.9(4.2)$ & $21.3(3.5)$ & $23.6(3.2)$ \\
\hline Diarrhea & 25.0 & $7.8(3.2)$ & $13.0(1.9)$ & $12.9(1.9)$ & $6.7(3.7)$ & $5.6(4.5)$ & $4.3(5.8)$ \\
\hline Other causes & - & 68.4 & 63.2 & 60.1 & 75.4 & 73.1 & 69.1 \\
\hline \multicolumn{8}{|c|}{$\begin{array}{l}\text { Proportional mortality by groups of } \\
\text { defined causes (ICD-10), < } 1 \text { yr. (\%) }\end{array}$} \\
\hline ARI & 70.0 & $10.2(6.9)$ & $8.9(7.9)$ & $9.7(9.7)$ & $8.7(8.0)$ & $11.1(6.3)$ & $10.7(6.5)$ \\
\hline Diarrhea & 20.0 & $8.1(2.5)$ & $9.3(2.2)$ & $15.4(1.3)$ & $6.4(3.1)$ & $5.0(4.0)$ & $4.7(4.3)$ \\
\hline Other causes & 10.0 & $81.7(0.1)$ & $81.8(0.1)$ & $74.9(0.1)$ & $84.9(0.1)$ & $83.9(0.1)$ & $84.6(0.1)$ \\
\hline
\end{tabular}

ARI: acute respiratory infection; CW: Central-West Region; ICD-10: International Classification of Diseases, 10th Revision; IMR: infant mortality rate;

MR: mortality rate; N: North Region; NE: Northeast Region; S: South Region; SE: Southeast Region.

* Victora \& Cesar 24;

** Ratio between the corresponding indicators: Guarani (numerator) and non-indigenous (Brazil or Region) indicated on heading (denominator);

*** Overall and sex-specific MR for Brazil and regions, as well as annual Guarani mortality rates, specific for ICD-10 chapters, were standardized by age and sex by the direct method, using as the reference the Brazilian inter-census population estimated by the Brazilian Institute of Geography and Statistics (Instituto Brasileiro de Geografia e Estatística - IBGE) for the year 2006, available at Health Informatics Department (Departamento de Informática do SUS - DATASUS. http://www.datasus.gov.br);

\# The overall Guarani MR was standardized by age and gender by the direct method, using as the reference the Brazilian population from the IBGE Census for 2000, available at DATASUS (http://www.datasus.gov.br). The standardized mortality rates for Brazil and Regions, presented by Inter-Agency Health Information Network (Rede Interagencial de Informações para a Saúde - RIPSA) for the year 2004, were also standardized for the same population (http://www.ibge. gov.br), allowing to compare the rates by ratios.

population in the various regions of the country, and the differences were larger in the South and Southeast. Meanwhile, the female Guarani MR exceeded the rates in the corresponding general Brazilian population by $10-60 \%$, with the largest differences in the North, Northeast, and CentralWest (Tables 1, 2 and 3).
The highest Guarani mortality rates were for diseases of the respiratory system (Chapter $\mathrm{X}$ ) and infectious and parasitic diseases (Chapter I). For these diseases, the Guarani rates were 1.9 (Southeast) to 3.3 (Northeast), and from 2.7 (Central-West) and 7.7 times higher (South) than the corresponding rates in the general popula- 
Guarani mortality indicators and comparisons with corresponding indicators for the non-indigenous population of Brazil and regions. Direct calculation and indirect estimates 2004

\begin{tabular}{|c|c|c|c|c|c|c|c|}
\hline \multirow[t]{2}{*}{ Indicators } & \multirow[t]{2}{*}{ Guarani } & \multicolumn{6}{|c|}{ Direct calculation and indirect estimates 2004 * } \\
\hline & & Brazil ** & $\mathbf{N} * *$ & NE ** & $\mathrm{CW} * *$ & $S * *$ & $\mathrm{SE} * *$ \\
\hline \multicolumn{8}{|c|}{ Overall and sex-specific MR (per 1,000$) \star \star \star, \#$} \\
\hline \multirow[t]{2}{*}{ Overall MR } & $5.3 * \star \star$ & $6.0 \#(0.8)$ & $6.1 \#(0.8)$ & $6.9 \#(0.7)$ & $5.6 \#(0.9)$ & $5.5 \#(0.9)$ & $5.8 \#(0.9)$ \\
\hline & $5.0 \#$ & & & & & & \\
\hline Male MR & 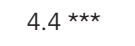 & - & - & - & - & - & - \\
\hline Female MR & $6.0^{\star \star \star}$ & - & - & - & - & - & - \\
\hline \multirow{2}{*}{\multicolumn{8}{|c|}{$\begin{array}{l}\text { Standardized specific MR by ICD-10 chapters } \\
(\text { per } 100,000) \star \star \star\end{array}$}} \\
\hline & & & & & & & \\
\hline MR, respiratory (Chap. X) & $124.3 * \star \star$ & - & - & - & - & - & - \\
\hline MR, infectious/ parasitic (Chap. I) & 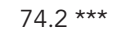 & - & - & - & - & - & - \\
\hline MR, digestive system (Chap. XI) & $7.5^{\star \star \star}$ & - & - & - & - & - & - \\
\hline MR, circulatory system (Chap. IX) & $42.3^{\star \star \star}$ & $157.3(0.3)$ & $72.8(0.6)$ & $119.8(0.4)$ & $142.4(0.3)$ & $190.0(0.2)$ & $188.5(0.2)$ \\
\hline MR, external causes (Chap. XX) & $49.3^{\star \star \star}$ & - & - & - & - & - & - \\
\hline MR, other causes & 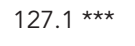 & - & - & - & - & - & - \\
\hline \multicolumn{8}{|l|}{ MR $<5$ yrs. and by specific causes } \\
\hline \multicolumn{8}{|l|}{ (per 1,000 live births) } \\
\hline $\mathrm{MR}<5$ yrs & 44.5 & $26.9(1.7)$ & $30.2(1.5)$ & $41.3(1.1)$ & $21.2(2.1)$ & $17.5(2.5)$ & $17.3(2.6)$ \\
\hline$M R<5$ yrs., ARI & 24.7 & - & - & - & - & - & - \\
\hline MR < 5 yrs., diarrhea & 7.4 & - & - & - & - & - & - \\
\hline MR $<5$ yrs., other causes & 12.4 & - & - & - & - & - & - \\
\hline Rates ratio ARI/diarrhea & 3.3 & - & - & - & - & - & - \\
\hline Rates ratio ARI/other causes & 2.0 & - & - & - & - & - & - \\
\hline \multicolumn{8}{|c|}{ IMR and components (per 1,000 live births) } \\
\hline IMR & 29.6 & $22.6(1.3)$ & $25.5(1.2)$ & $33.9(0.9)$ & $18.7(1.6)$ & $15.0(2.0)$ & $14.9(2.0)$ \\
\hline Early neonatal IMR (0-6 days) & 4.9 & $11.5(0.4)$ & $12.8(0.4)$ & $17.6(0.3)$ & $9.2(0.5)$ & $7.4(0.7)$ & $7.6(0.6)$ \\
\hline Late neonatal IMR (7-27 days) & - & 3.5 & 3.5 & 4.4 & 3.3 & 2.6 & 2.7 \\
\hline Post-neonatal IMR (28-364 days) & 24.7 & $7.6(3.3)$ & $9.2(2.7)$ & $12.0(2.1)$ & $6.2(4.0)$ & $5.0(4.9)$ & $4.6(5.4)$ \\
\hline \multicolumn{8}{|c|}{ IMR by selected causes (per 1,000 live births) } \\
\hline IMR from ARI & 17.3 & - & - & - & - & - & - \\
\hline IMR from diarrhea & 4.9 & - & - & - & - & - & - \\
\hline IMR from other causes & 7.4 & - & - & - & - & - & - \\
\hline Rates ratio ARI/diarrhea & 3.5 & - & - & - & - & - & - \\
\hline Rates ratio ARI/other causes & 2.3 & - & - & - & - & - & - \\
\hline \multicolumn{8}{|l|}{ Age-proportional mortality (\%) } \\
\hline$<5$ yrs. & 48.6 & $6.2(7.8)$ & $14.4(3.4)$ & $8.9(5.5)$ & $7.5(6.5)$ & $4.3(11.3)$ & $4.4(11.0)$ \\
\hline $1-4 \mathrm{yrs}$ & 16.2 & - & - & - & - & - & - \\
\hline$<1 \mathrm{yr}$ & 32.4 & - & - & - & - & - & - \\
\hline Early neonatal (0-6 days) & 16.7 & $50.9(0.3)$ & $50.1(0.3)$ & $51.8(0.3)$ & $49.1(0.3)$ & $49.5(0.3)$ & $51.0(0.3)$ \\
\hline Late neonatal (7-27 days) & - & 15.5 & 13.8 & 13.0 & 17.7 & 17.2 & 17.8 \\
\hline Post-neonatal (28-364 days) & 83.3 & $33.6(2.5)$ & $36.1(2.3)$ & $35.3(2.4)$ & $33.2(2.5)$ & $33.3(2.5)$ & $31.2(2.7)$ \\
\hline \multicolumn{8}{|c|}{ Proportion of deaths from ill-defined causes (\%) } \\
\hline Overall population & 15.8 & $12.4(1.3)$ & $20.8(0.8)$ & $23.7(0.7)$ & $5.8(2.7)$ & $6.2(2.5)$ & 8.5 (1.9) \\
\hline$<5$ yrs. & 22.2 & - & - & - & - & - & - \\
\hline $1-4 \mathrm{yrs}$ & 33.3 & - & - & - & - & - & - \\
\hline$<1$ yr. & 16.7 & - & - & - & - & - & - \\
\hline
\end{tabular}

(continues) 
Table 2 (continued)

\begin{tabular}{|c|c|c|c|c|c|c|c|}
\hline \multirow[t]{2}{*}{ Indicators } & \multirow[t]{2}{*}{ Guarani } & \multicolumn{6}{|c|}{ Direct calculation and indirect estimates 2004 * } \\
\hline & & Brazil ** & $\mathbf{N} * *$ & NE ** & $\mathrm{CW} * *$ & $\mathrm{~S} * *$ & SE ** \\
\hline \multirow{2}{*}{\multicolumn{8}{|c|}{$\begin{array}{l}\text { Proportional mortality by groups of defined causes } \\
(I C D-10) \text { in population (\%) }\end{array}$}} \\
\hline & & & & & & & \\
\hline Respiratory (Chap. X) & 40.6 & $11.4(3.6)$ & $11.1(3.7)$ & $9.5(4.3)$ & $10.1(4.0)$ & $11.8(3.4)$ & $12.2(3.4)$ \\
\hline Infectious/ parasitic (Chap. I) & 18.8 & $5.1(3.7)$ & $7.3(2.6)$ & $6.0(3.1)$ & $5.5(3.4)$ & $4.0(4.7)$ & $4.9(3.8)$ \\
\hline Digestive (Chap. XI) & 12.5 & - & - & - & - & - & - \\
\hline Circulatory (Chap. IX) & 9.4 & $31.8(0.3)$ & $24.3(0.4)$ & $30.9(0.3)$ & $30.8(0.3)$ & $33.1(0.3)$ & $32.7(0.3)$ \\
\hline External causes (Chap. XX) & 9.4 & $14.2(0.7)$ & $18.9(0.5)$ & $15.5(0.6)$ & $17.8(0.5)$ & $12.6(0.7)$ & $13.3(0.7)$ \\
\hline Other causes & 9.4 & - & - & - & - & - & - \\
\hline \multirow{2}{*}{\multicolumn{8}{|c|}{$\begin{array}{l}\text { Proportional mortality by groups of defined causes } \\
(\text { ICD-10), < } 5 \text { yrs. (\%) }\end{array}$}} \\
\hline & & & & & & & \\
\hline ARI & 71.4 & $5.8(12.3)$ & $7.4(9.6)$ & $5.3(13.5)$ & $6.2(11.5)$ & $5.2(13.7)$ & $5.7(12.5)$ \\
\hline Diarrhea & 21.4 & $4.0(5.4)$ & $4.9(4.4)$ & $6.2(3.5)$ & $3.9(5.5)$ & $2.1(10.2)$ & $1.9(11.3)$ \\
\hline Other causes & 7.1 & $90.2(0.1)$ & $87.7(0.1)$ & $88.5(0.1)$ & $89.9(0.1)$ & $92.7(0.1)$ & $92.4(0.1)$ \\
\hline \multirow{2}{*}{\multicolumn{8}{|c|}{$\begin{array}{l}\text { Proportional mortality by groups of defined causes } \\
\text { (ICD-10), 1-4 yrs. (\%) }\end{array}$}} \\
\hline & & & & & & & \\
\hline ARI & 75.0 & - & - & - & - & - & - \\
\hline Diarrhea & 25.0 & - & - & - & - & - & - \\
\hline Other causes & - & - & - & - & - & - & - \\
\hline \multirow{2}{*}{\multicolumn{8}{|c|}{$\begin{array}{l}\text { Proportional mortality by groups of defined causes } \\
(\text { ICD-10), < } 1 \text { yr. (\%) }\end{array}$}} \\
\hline & & & & & & & \\
\hline ARI & 70.0 & - & - & - & - & - & - \\
\hline Diarrhea & 20.0 & - & - & - & - & - & - \\
\hline Other causes & 10.0 & - & - & - & - & - & - \\
\hline
\end{tabular}

ARI: acute respiratory infection; CW: Central-West region; ICD-10: International Classification of Diseases, 10th Revision; IMR: infant mortality rate; MR: mortality rate; N: North region; NE: Northeast region; S: South region; SE: Southeast region.

* RIPSA 23;

** Ratio between the corresponding indicators: Guarani (numerator) and non-indigenous (Brazil or Region) indicated on heading (denominator);

*** Overall and sex-specific MR for Brazil and regions, as well as annual Guarani mortality rates, specific for ICD-10 chapters, were standardized by age and sex by the direct method, using as the reference the Brazilian inter-census population estimated by the Brazilian Institute of Geography and Statistics (Instituto Brasileiro de Geografia e Estatística - IBGE) for the year 2006, available at Health Informatics Department (Departamento de Informática do SUS - DATASUS. http://www.datasus.gov.br);

\# The overall Guarani MR was standardized by age and sex by the direct method, using as the reference the Brazilian population from the IBGE Census for 2000, available at DATASUS (http://www.datasus.gov.br). The standardized mortality rates for Brazil and Regions, presented by Inter-Agency Health Information Network (Rede Interagencial de Informações para a Saúde - RIPSA) for the year 2004, were also standardized for the same population (http://www.ibge.gov.br), allowing to compare the rates by ratios.

tion in the various regions of Brazil, respectively. Meanwhile, for diseases of the circulatory system (Chapter IX) and external causes (Chapter XX), the MR were higher in the general Brazilian population, except in the comparison of deaths from external causes in the South, where the Guarani MR was 3.6 times higher than the corresponding rate in the general population.

The Guarani MR in under-five children was $44.5 / 1,000$ live births, or $10 \%$ higher than the MR in the Northeast, $50 \%$ higher than in the North, and 2.1 to 2.6 times higher than the corresponding MR in the other regions, estimated according to the RIPSA. As compared to the MR calculated directly from DATASUS data for the year 2006, the size of the rates ratios in this age bracket increased, with Guarani MR ranging from 1.9 times higher in the North to 2.8 times higher in the South when compared to the general population.

The specific Guarani MR from ARI in underfive children was the highest among the specific causes of mortality in this age group, and was 3.3 and 2.0 times higher than the MR from diarrhea and other causes, respectively. As compared to the equivalent MR obtained from direct calculation for Brazilian children as a whole, the Guarani MR from ARI was 15.4 times (North) to 41.2 times (South) higher than the MR for all Brazilian chil- 
Guarani mortality indicators and comparisons with corresponding indicators for the non-indigenous population of Brazil and regions. Direct calculation 2006.

\begin{tabular}{|c|c|c|c|c|c|c|c|}
\hline \multirow[t]{2}{*}{ Indicators } & \multirow[t]{2}{*}{ Guarani } & \multicolumn{6}{|c|}{ Direct calculation 2006 * } \\
\hline & & Brazil ** & $\mathbf{N} * *$ & NE ** & $\mathrm{CW} * *$ & $S^{* *}$ & $\mathrm{SE} * *$ \\
\hline \multicolumn{8}{|l|}{ Overall and sex-specific MR } \\
\hline \multicolumn{8}{|l|}{$($ per 1,000$) * \star *, \#$} \\
\hline \multirow[t]{2}{*}{ Overall MR } & $5.3^{\star \star \star *}$ & $5.5(1.0)$ & $4.8^{\star \star \star}(1.1)$ & $5.3^{\star \star \star}(1.0)$ & $5.6^{\star \star \star}(0.9)$ & $6.3^{\star \star \star *}(0.8)$ & $6.4^{\star \star \star *}(0.8)$ \\
\hline & $5.0 \#$ & & & & & & \\
\hline Male MR & $4.4^{\star \star \star}$ & $6.5(0.7)$ & $5.9^{\star \star \star *}(0.7)$ & $6.2^{\star \star \star}(0.7)$ & 7.0 *** $(0.6)$ & $7.3^{\star \star \star *}(0.6)$ & $7.5^{\star \star * \star}(0.6)$ \\
\hline Female MR & $6.0 * \star *$ & $4.6(1.3)$ & $3.8^{* \star \star}(1.6)$ & $4.5^{\star \star \star \star}(1.3)$ & $4.3^{* \star \star}(1.4)$ & $5.3^{\star \star \star}(1.1)$ & $5.3 * \star *(1.1)$ \\
\hline \multicolumn{8}{|l|}{ Standardized specific MR by } \\
\hline \multicolumn{8}{|l|}{ 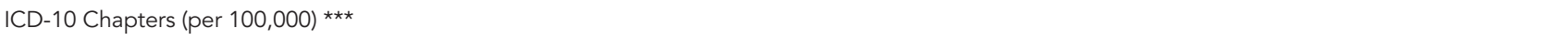 } \\
\hline MR , respiratory (Chap. X) & $124.3^{\star \star \star *}$ & $55.1(2.3)$ & $42.6^{\star \star \star}(2.9)$ & $37.6^{\star \star \star}(3.3)$ & $58.3^{* \star \star}(2.1)$ & $41.0 * \star \star(3.0)$ & $64.2^{\star \star \star}(1.9)$ \\
\hline MR, infectious/ parasitic (Chap. I) & 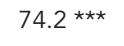 & $24.9(3.0)$ & $25.6^{\star \star \star}(2.9)$ & $24.9^{* \star \star}(3.0)$ & 27.9 *** $(2.7)$ & $9.6 * * \star(7.7)$ & 25.0 *** (3.0) \\
\hline MR, digestive system (Chap. XI) & $7.5 * \star \star$ & $27.8(0.3)$ & $21.7^{\star \star \star}(0.3)$ & $25.2^{\star \star \star}(0.3)$ & 28.4 *** $(0.3)$ & $13.3^{\star \star \star}(0.6)$ & $30.2^{\star \star \star}(0.2)$ \\
\hline MR, circulatory system (Chap. IX) & 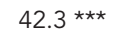 & $162.1(0.3)$ & $116.8^{\star \star \star}(0.4)$ & $144.8^{* \star \star}(0.3)$ & $177.4^{\star \star \star}(0.2)$ & $106.9^{* \star \star}(0.4)$ & $173.3^{\star \star \star}(0.2)$ \\
\hline MR, external causes (Chap. XX) & $49.3 * \star \star$ & $68.7(0.7)$ & $65.7^{\star \star \star}(0.8)$ & $68.3^{\star \star \star}(0.7)$ & $77.1 \star \star \star ~(0.6)$ & 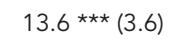 & $67.0 * * \star(0.7)$ \\
\hline MR, other causes & 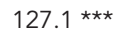 & $213.7(0.6)$ & 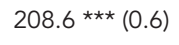 & 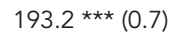 & $196.0 * \star \star(0.6)$ & $123.4^{* \star \star}(1.0)$ & $224.9^{\star \star \star *}(0.6)$ \\
\hline \multicolumn{8}{|l|}{ MR $<5$ yrs. and by specific causes } \\
\hline \multicolumn{8}{|l|}{ (per 1,000 live births) } \\
\hline $\mathrm{MR}<5 \mathrm{yrs}$ & 44.5 & $19.3(2.3)$ & $23.3(1.9)$ & $22.5(2.0)$ & $18.4(2.4)$ & $15.8(2.8)$ & $17.0(2.6)$ \\
\hline $\mathrm{MR}<5$ yrs., ARI & 24.7 & $1.1(22.5)$ & $1.6(15.4)$ & $1.3(19.0)$ & $1.0(24.7)$ & $0.6(41.2)$ & $1.0(24.7)$ \\
\hline MR < 5 yrs., diarrhea & 7.4 & $0.70(10.6)$ & $0.90(8.2)$ & $1.4(5.3)$ & $0.60(12.3)$ & $0.20(37.0)$ & $0.30(24.7)$ \\
\hline MR $<5$ yrs., other causes & 12.4 & $17.5(0.7)$ & $20.8(0.6)$ & $19.9(0.6)$ & $16.8(0.7)$ & $15.0(0.8)$ & $15.8(0.8)$ \\
\hline Rates ratio ARI/diarrhea & 3.3 & 1.6 & 1.8 & 0.9 & 1.7 & 3.0 & 3.3 \\
\hline Rates ratio ARI/other causes & 2.0 & 0.1 & 0.1 & 0.1 & 0.1 & 0.0 & 0.1 \\
\hline \multicolumn{8}{|c|}{ IMR and components (per 1,000 live births) } \\
\hline IMR & 29.6 & $16.4(1.8)$ & $19.5(1.5)$ & $19.3(1.5)$ & $15.5(1.9)$ & $13.3(2.2)$ & $14.5(2.0)$ \\
\hline Early neonatal IMR (0-6 days) & 4.9 & $8.6(0.6)$ & $10.1(0.5)$ & $10.4(0.5)$ & $7.8(0.6)$ & $6.9(0.7)$ & $7.4(0.7)$ \\
\hline Late neonatal IMR (7-27 days) & - & 2.5 & 2.7 & 2.5 & 2.5 & 2.3 & 2.5 \\
\hline Post-neonatal IMR (28-364 days) & 24.7 & $5.3(4.7)$ & $6.7(3.7)$ & $6.3(3.9)$ & $5.1(4.8)$ & $4.2(5.9)$ & $4.6(5.4)$ \\
\hline \multicolumn{8}{|l|}{ IMR by selected causes } \\
\hline \multicolumn{8}{|l|}{ (per 1,000 live births) } \\
\hline IMR from ARI & 17.3 & $0.8(21.6)$ & $1.2(14.4)$ & $0.9(19.2)$ & $0.7(24.7)$ & $0.4(43.3)$ & $0.7(24.7)$ \\
\hline IMR from diarrhea & 4.9 & $0.5(9.8)$ & $0.7(7.0)$ & $1.1(4.5)$ & $0.4(12.3)$ & $0.2(24.5)$ & $0.2(24.5)$ \\
\hline IMR from other causes & 7.4 & $15.1(0.5)$ & $17.7(0.4)$ & $17.2(0.4)$ & $14.4(0.5)$ & $12.7(0.6)$ & $13.7(0.5)$ \\
\hline Rates ratio ARI/diarrhea & 3.5 & 1.4 & 1.7 & 0.8 & 1.6 & 2.8 & 3.0 \\
\hline Rates ratio ARI/other causes & 2.3 & 0.1 & 0.1 & 0.1 & 0.1 & 0.0 & 0.1 \\
\hline \multicolumn{8}{|l|}{ Age-proportional mortality (\%) } \\
\hline$<5$ yrs. & 48.6 & $5.5(8.8)$ & $13.2(3.7)$ & $7.8(6.2)$ & $6.5(7.5)$ & $3.7(13.1)$ & $3.9(12.5)$ \\
\hline $1-4 \mathrm{yrs}$ & 16.2 & $0.8(20.3)$ & $2.1(7.7)$ & $1.1(14.7)$ & $1.0(16.2)$ & $0.6(27.0)$ & $0.5(32.4)$ \\
\hline$<1 \mathrm{yr}$ & 32.4 & $4.7(6.9)$ & $11.1(2.9)$ & $6.7(4.8)$ & $5.5(5.9)$ & $3.1(10.5)$ & $3.4(9.5)$ \\
\hline Early neonatal (0-6 days) & 16.7 & $51.9(0.3)$ & $54.0(0.3)$ & $51.0(0.3)$ & $51.6(0.3)$ & $50.7(0.3)$ & $52.2(0.3)$ \\
\hline Late neonatal (7-27 days) & - & 13.8 & 13.1 & 17.1 & 16.9 & 16.5 & 15.2 \\
\hline Post-neonatal (28-364 days) & 83.3 & $34.3(2.4)$ & $32.9(2.5)$ & $31.8(2.6)$ & $31.5(2.6)$ & $32.9(2.5)$ & $32.5(2.6)$ \\
\hline
\end{tabular}

(continues) 
Table 3 (continued)

\begin{tabular}{|c|c|c|c|c|c|c|c|}
\hline \multirow[t]{2}{*}{ Indicators } & \multirow[t]{2}{*}{ Guarani } & \multicolumn{6}{|c|}{ Direct calculation 2006 * } \\
\hline & & Brazil ** & $\mathbf{N} * *$ & NE ** & $\mathrm{CW} \star \star$ & $S * \star$ & $\mathrm{SE} * \star$ \\
\hline \multicolumn{8}{|c|}{ Proportion of deaths from ill-defined } \\
\hline \multicolumn{8}{|c|}{ causes $(\%)$} \\
\hline Overall population & 15.8 & $8.3(1.9)$ & $15.0(1.1)$ & $9.5(1.7)$ & $4.9(3.2)$ & $5.8(2.7)$ & $8.2(1.9)$ \\
\hline$<5$ yrs. & 22.2 & $5.2(4.3)$ & $9.7(2.3)$ & $4.7(4.7)$ & $3.4(6.5)$ & $4.0(5.6)$ & $4.8(4.6)$ \\
\hline $1-4$ yrs. & 33.3 & $9.0(3.7)$ & $17.5(1.9)$ & $8.3(4.0)$ & $5.9(5.6)$ & $5.5(6.1)$ & $8.0(4.2)$ \\
\hline$<1 \mathrm{yr}$ & 16.7 & $4.6(3.6)$ & $8.1(2.1)$ & $4.1(4.1)$ & $2.9(5.8)$ & $3.8(4.4)$ & $4.3(3.9)$ \\
\hline \multicolumn{8}{|c|}{ Proportional mortality by groups of defined } \\
\hline \multicolumn{8}{|c|}{ causes (ICD-10) in population (\%) } \\
\hline Respiratory (Chap. X) & 40.6 & $10.9(3.7)$ & $9.7(4.2)$ & $8.7(4.7)$ & $9.9(4.1)$ & $11.8(3.4)$ & $11.9(3.4)$ \\
\hline Infectious/ parasitic (Chap. I) & 18.8 & $4.9(3.8)$ & $6.9(2.7)$ & $5.5(3.4)$ & $5.3(3.5)$ & $4.0(4.7)$ & $4.7(4.0)$ \\
\hline Digestive (Chap. XI) & 12.5 & $5.5(2.3)$ & $5.0(2.5)$ & $5.4(2.3)$ & $5.3(2.4)$ & $5.2(2.4)$ & $5.7(2.2)$ \\
\hline Circulatory (Chap. IX) & 9.4 & $32.0(0.3)$ & $24.8(0.4)$ & $32.7(0.3)$ & $30.8(0.3)$ & $31.9(0.3)$ & $32.6(0.3)$ \\
\hline External causes (Chap. XX) & 9.4 & $13.6(0.7)$ & $19.6(0.5)$ & $14.6(0.6)$ & $17.2(0.5)$ & $12.5(0.8)$ & $12.3(0.8)$ \\
\hline Other causes & 9.4 & $33.1(0.3)$ & $33.9(0.3)$ & $33.2(0.3)$ & $31.4(0.3)$ & $34.5(0.3)$ & $32.8(0.3)$ \\
\hline \multirow{2}{*}{\multicolumn{8}{|c|}{$\begin{array}{l}\text { Proportional mortality by groups of defined } \\
\text { causes (ICD-10), < } 5 \text { yrs. (\%) }\end{array}$}} \\
\hline & & & & & & & \\
\hline ARI & 71.4 & $5.7(12.5)$ & $7.0(10.2)$ & $5.8(12.3)$ & $5.4(13.2)$ & $4.0(17.9)$ & $5.8(12.3)$ \\
\hline Diarrhea & 21.4 & $3.5(6.1)$ & $3.8(5.6)$ & $6.0(3.6)$ & $3.3(6.5)$ & $1.3(16.5)$ & $1.6(13.4)$ \\
\hline Other causes & 7.1 & $90.7(0.1)$ & $89.1(0.1)$ & $88.2(0.1)$ & $91.3(0.1)$ & $94.7(0.1)$ & $92.6(0.1)$ \\
\hline \multirow{2}{*}{\multicolumn{8}{|c|}{$\begin{array}{l}\text { Proportional mortality by groups of defined } \\
\text { causes (ICD-10), 1-4 yrs. (\%) }\end{array}$}} \\
\hline & & & & & & & \\
\hline ARI & 75.0 & $12.3(6.1)$ & $13.3(5.6)$ & $12.4(6.0)$ & $12.0(6.3)$ & $8.2(9.1)$ & $13.3(5.6)$ \\
\hline Diarrhea & 25.0 & $4.8(5.2)$ & $5.6(4.5)$ & $7.4(3.4)$ & $6.8(3.7)$ & $2.0(12.5)$ & $2.3(10.9)$ \\
\hline Other causes & - & 82.9 & 81.1 & 80.2 & 81.2 & 89.8 & 84.4 \\
\hline \multirow{2}{*}{\multicolumn{8}{|c|}{$\begin{array}{l}\text { Proportional mortality by groups of defined } \\
\text { causes (ICD-10), < } 1 \text { yr. (\%) }\end{array}$}} \\
\hline & & & & & & & \\
\hline ARI & 70.0 & 4.5 (15.6) & $5.8(12.1)$ & $4.6(15.2)$ & $4.2(16.7)$ & 3.2 (21.9) & 4.5 (15.6) \\
\hline Diarrhea & 20.0 & $3.3(6.1)$ & $3.5(5.7)$ & $5.8(3.4)$ & $2.7(7.4)$ & $1.2(16.7)$ & $1.5(13.3)$ \\
\hline Other causes & 10.0 & $92.1(0.1)$ & $90.7(0.1)$ & $89.6(0.1)$ & $93.2(0.1)$ & $95.6(0.1)$ & $94.0(0.1)$ \\
\hline
\end{tabular}

ARI: acute respiratory infection; CW: Central-West region; ICD-10: International Classification of Diseases, 10th Revision; IMR: infant mortality rate; MR: mortality rate; N: North region; NE: Northeast region; S: South region; SE: Southeast region.

* DATASUS (http://www.datasus.gov.br, accessed on 17/Nov/2009);

** Ratio between the corresponding indicators: Guarani (numerator) and non-indigenous (Brazil or Region) indicated on heading (denominator);

*** Overall and sex-specific MR for Brazil and regions, as well as annual Guarani mortality rates, specific for ICD-10 chapters, were standardized by age and sex by the direct method, using as the reference the Brazilian inter-census population estimated by the Brazilian Institute of Geography and Statistics (Instituto Brasileiro de Geografia e Estatística - IBGE) for the year 2006, available at Health Informatics Department (Departamento de Informática do SUS - DATASUS. http://www.datasus.gov.br);

\# The overall Guarani MR was standardized by age and sex by the direct method, using as the reference the Brazilian population from the IBGE Census for 2000, available at DATASUS (http://www.datasus.gov.br). The standardized mortality rates for Brazil and Regions, presented by Inter-Agency Health Information Network (Rede Interagencial de Informações para a Saúde - RIPSA) for the year 2004, were also standardized for the same population (http://www.ibge.gov.br), allowing to compare the rates by ratios.

dren according to region. The rates ratios were also high for diarrhea in the South and Southeast, while the MR due to other causes in Brazilian children as a whole exceeded the corresponding Guarani MR in all the regions.

The Guarani infant mortality rate (IMR) was $29.6 / 1,000$ live births, exceeding by $10-30 \%$ the estimated IMR for the South, Southeast, and
Central-West for 10 years 24. As compared to the IMR calculated according to the RIPSA, the Guarani IMR was only slightly lower than that reported for the Northeast (33.9/1000), and was $20 \%$ higher than that of the North and twice as high as the South and Southeast. As for the IMR calculated directly from the DATASUS data, the Guarani IMR even exceeded the IMR for the 
Northeast, with slightly higher rates ratios, and the difference was even higher in the South. Unlike the composition of IMR for Brazilian children in general, the magnitude of Guarani postneonatal IMR was five times greater than early neonatal IMR, exceeding by 2.1 times (Northeast) and 5.4 times (Southeast) the corresponding IMR estimated by the RIPSA and by 3.7 times (North) and 5.9 times (South) the rates calculated directly from DATASUS.

The IMR from acute respiratory infections was the highest among specific causes of infant mortality in the Guarani, and was 3.5 and 2.3 times higher than the Guarani IMR from diarrhea and other causes, respectively. Guarani IMR from ARI were 2.9 times (Northeast) to 7.5 times (Central-West) higher than those of regions of Brazil ten years earlier 24. This same comparison for diarrhea showed a higher diarrhea-specific IMR in the Northeast of Brazil as compared to the diarrhea-specific IMR among the Guarani. The IMR ratios due to specific selected causes using direct calculation from DATASUS showed higher IMR for the Guarani for all causes, especially ARI, whose ratios ranged from 14.4 (North) to 43.3 times (South).

The proportion of deaths in Guarani infants ( $<1$ year) was comparable to the 50 -and-older age group (32.4\%). The proportions were also the same comparing the 1-4-year and 20-49-year groups (16.2\%). The lowest proportion of deaths occurred from 5 to 19 years of age $(2.7 \%)$. Deaths in under-five Guarani children comprised nearly half $(48.6 \%)$ of all deaths in the Guarani population (Tables 1, 2 and 3).

The proportions of deaths in Guarani infants $(<1$ year) and under-fives exceeded those for the general Brazilian population in all regions of the country, regardless of the data source used for the comparison. In under-five Guarani children, the proportion of deaths was closest to the estimate for the North of Brazil according to the RIPSA data, but it was still 3.4 times higher, reaching 11 times higher than the proportions in the South and Southeast. In infants ( $<1$ year), the Guarani proportion of deaths was 2.9 times (North) to 10.5 times (South) higher than the corresponding proportions according to direct calculations using DATASUS data for Brazilian children. Of all the Guarani infant deaths, $83.3 \%$ occurred in the post-neonatal period, with twice the corresponding rates in all regions of the country, regardless of the data sources, except for the Northeast for the 1995-97 three-year period 24 , which magnitude was exceeded by $70 \%$ of the proportion observed among the Guarani.

The proportion of deaths from ill-defined causes (ICD Chapter XVIII) was high among the Guarani, especially in the 1-4-year bracket (33.3\%) (Tables 1, 2 and 3). In nearly all of the comparisons, the proportions of deaths from ill-defined causes among the Guarani exceeded the corresponding proportions in the general Brazilian population. Excluding these causes, respiratory diseases (Chapter X: $40.6 \%$ ) were the principal causes of death among the Guarani, followed by infectious and parasitic diseases (Chapter I: 18.8\%), while among the general Brazilian population the main causes were diseases of the circulatory system (Chapter IX), external causes (Chapter XX), and other causes. In Guarani under-fives, $71.4 \%$ of the deaths were due to ARI and $21.4 \%$ to diarrheas, exceeding by at least 9.6 times the proportions of deaths from ARI (North) and by at least 3.5 times those from diarrhea (Northeast) for children under five in Brazil, estimated from RIPSA data. ARI and diarrhea jointly accounted for $100 \%$ of the causes of Guarani mortality in the 1-4-year group and $90 \%$ of infant mortality (<1 year) (Tables 1, 2 and 3).

\section{Discussion}

A first point for discussion is the coverage rate for vital events analyzed in this study. In the South and Southeast of Brazil, the health workers from the EMSI routinely visit in the villages, so it is unlikely that deaths or births could occur without their knowledge, thus indicating the probable recording of all such events in the current study. Even so, it is possible that some early neonatal deaths were inadequately classified as stillbirths, since approximately $30 \%$ of the births occur at home.

In the case of small populations such as indigenous groups, it is recommended that deaths be analyzed using means from multipleyear periods, in order to avoid instability in the rates 13,16 . Since our data were collected over the course of only 14 months, this procedure was not possible. However, the Guarani population analyzed here greatly exceeds the mean population of the majority of the indigenous ethnic groups in Brazil, since approximately half of the country's 225 indigenous peoples have fewer than 500 individuals each 25 . In addition, the comparison of direct and indirect methods for calculating Kaiabi (an indigenous group from Central Brazil) demographic indicators showed that it is possible, even in small-scale populations, to produce consistent mortality indicators, as long as there are accurate records of vital events 26 .

A potential limitation to analyzing causes of death might be the high proportion of ill- 
defined causes. Still, even if all the deaths from illdefined causes were combined with the second most common cause of death (diarrhea), the sum would still be less than the proportional mortality from respiratory diseases, thus confirming the latter as the principal cause of death in the Guarani. As for defined causes of death, those due to ALRI in under-five children were confirmed through the review of hospital charts, so the impact of possible misclassifications would probably be negligible.

In recent decades, Brazil has witnessed a steady drop in fertility and mortality rates, accompanied by population aging 5 . The proportion of deaths at more advanced ages, especially from cardiovascular diseases and cancer, has increased, while the infant and under-five mortality rates have declined, with early neonatal deaths (due to perinatal causes) now predominating, along with a reduction in infectious causes. There has also been a drop in ill-defined causes of death. Meanwhile, the country has seen intense urbanization and improved access to basic sanitation, health services, and education. Despite these improvements, the Brazilian population's current morbidity and mortality pattern is still marked by socioeconomic and environmental determinants and regional and ethnic/racial inequalities 5,6,7,8,9,10,27.

In general, indigenous groups in Brazil have experienced rapid demographic recovery, with high population growth rates 28 . The Guarani age structure is similar to that of other indigenous peoples living in rural areas of Brazil 17, whereby the pyramid shape reflects high birth and death rates, low median age, and low life expectancy at birth 14,17,29. Some studies confirm the high Guarani fertility rate and a process of demographic recovery which have combined with moderate mortality rates 29 to result in the observed demographic profile.

Importantly, $20.2 \%, 52.1 \%$, and $4 \%$ of the Guarani were under five, under 15, and 65 years or older, respectively, while the corresponding proportions in the general Brazilian population were $8.9 \%, 28 \%$, and $6.3 \%$ in 2006 (http://www. datasus.gov.br, accessed on $17 /$ Nov/2009). The population pyramid in the North of Brazil is the most similar to that of the Guarani, but still with a smaller contingent of children and young people. In the South and Southeast, where the Guarani villages are located, the mean proportions under five, under 15, and 65 years or older in the general population were $7.9 \%, 25.3 \%$, and $7.1 \%$, respectively, thus characterizing an older population 25 , as corroborated by the mean aging index for the Brazilian population (33.9 $\geq 60$ years $/ 100$ $<15$ years) estimated by the RIPSA 23, 3.4 times higher than the Guarani rate $(9.8 \geq 60$ years $/ 100$ $<15$ years).

The male-female ratio in the Guarani (108.8) was similar to (but higher than) the corresponding ratio for all indigenous peoples living on indigenous lands in Brazil according to the 2000 national census (107.0) 17, and was closer to the indigenous male-female ratio in the North (109.6) and higher than the corresponding ratios in Southeast (102.0) and South (100.0) (6.7\% and $8.8 \%$, higher, respectively). The Guarani discrepancy in relation to the sex ratios in rural indigenous peoples in the South and Southeast may be due to demographic differences among the indigenous groups living in these areas or to the partial capture of demographic data for these indigenous peoples in the 2000 Census.

Many populations show more births of boys than girls 29 , while among the Guarani this ratio was the opposite. This female surplus could be explained by random sex fluctuations in births, or by random or non-random differential stillbirth rates between the sexes 14,26,29. Nevertheless, there were more boys in the 0-4-year age group (118.5), which could be explained by higher female mortality in the first years of life, as demonstrated by the sex-specific mortality rates.

The North of Brazil was the only region with a male majority in the sex ratio (102.2) in $2004{ }^{23}$. The larger number of women in the general Brazilian population has been attributed to excess male mortality resulting from higher risk related to external causes and cardiovascular diseases and lower access to preventive health care. Meanwhile, in the North the larger number of men is attributed to immigration for socioeconomic reasons, particularly work opportunities 23 . However, the factors that explain the male majority in the Guarani sex ratio appear to diverge from those in the North, since the effect of migration on the Guarani indicator would only be explained by major emigration of women to the cities or out of the specific indigenous territory. This is unlikely, although there are other Guarani villages outside the territory, and Guarani families are known to move between villages.

The Guarani sex ratio curve according to age groups appears to reflect diverse experiences in different population cohorts, where the ratio in younger age brackets reflects the excess female Guarani mortality at earlier ages, compensated for by excess male mortality in adulthood. Meanwhile, the ratio in the elderly might reflect excess female mortality in this population cohort during the childbearing period of these women, when they were still young. In addition, it is possible that the sex ratios in older age brackets are dis- 
torted by upward rounding of ages in older individuals who lack birth records, which could affect the sex counts differently.

The overall Guarani MR was half-way between the rates for various indigenous peoples in Amazonia (3.3/1,000 in the Suyá 13 to 10.0/1,000 in the Hupd'ah 30 ) and was close the rates for the general population in all regions of Brazil. The fact that it was similar to the Northeast and higher than the North, based on direct comparison with data from DATASUS, may reflect problems of underreporting of deaths in the regions, since according to the comparison with indirect RIPSA indicators 23, Guarani mortality was also lower than those observed in these regions.

The female Guarani MR exceeded the male rate, although the difference was not statistically significant. This pattern could be attributed to higher female mortality due to respiratory causes, particularly in under-five children, possibly due to the greater exposure of girls to environmental determinants of ALRI, like the smoke and soot produced by burning firewood to cook food 20,21.

The standardized male Guarani MR was lower than the Brazilian rate in all regions, including the North and Northeast, where under-recording of deaths supposedly occurs, and was even $40 \%$ lower than male mortality in the South and Southeast. On the other hand, female Guarani mortality was at least $10 \%$ higher than the overall Brazilian female rate in all regions of the country. These differences result from probable inequalities in exposure to mortality risks from different causes according to sex and age group in the Guarani and general Brazilian populations. The Guarani studied here are more exposed to adverse socioeconomic and environmental conditions, expressed by greater risk of death related to maternal and child health factors and infectious causes and lower risk related to chronic noncommunicable diseases and external causes.

The Guarani under-five MR was high and mainly because ARI. The MR in this age bracket exceeded by at least $10 \%$ the corresponding mortality rate in Brazilian children in general and by 2.5 times the mortality rate for children in the South and Southeast of the country. The Guarani infant mortality rate was equal to the mean for indigenous peoples in the Xingu and intermediate in relation to the IMR for other Brazilian indigenous peoples (15.2/1,000 in the Kaiabi 14 and 116.3/1,000 in the Hupd'ah 30). The current Guarani IMR has been $10 \%$ to $30 \%$ higher than the IMR in the South, Southeast, and Central-West of Brazil for 12 years 24. In the comparison with more current infant mortality rates, the Guarani IMR was only exceeded (by 10\%) by that of the Northeast in 2004, while the Guarani rate exceed- ed by at least $20 \%$ the IMR for the other regions, including the North, and it was double the IMR of the South and Southeast 23 .

While the Guarani post-neonatal IMR was five times of the Guarani early neonatal IMR, the opposite was true for Brazilian children in general, where early neonatal IMR was $50 \%$ to $60 \%$ greater than post-neonatal IMR. Early neonatal IMR in the Guarani was at least 30\% lower than the corresponding IMR in the various regions of the country, while post-neonatal IMR in the Guarani exceeded the corresponding IMR in Brazilian children in general by 2.1 to 5.9 , with the largest differences in relation to the South and Southeast. The principal causes of infant deaths in the Guarani were acute respiratory infections, followed by diarrhea, which explains the high post-neonatal IMR, the probable results of adverse health and living conditions.

Approximately half of the Guarani deaths occurred under five years of age and $34.4 \%$ in infants ( $<1$ year), and these proportions were much higher than those in the general population. The proportion of deaths from ill-defined causes in the Guarani population was high, especially in the 1-4-year and under-1-year brackets. In children under five years, the proportion was 2.3 to 6.5 times higher than the corresponding proportions in the Brazilian regions, where the proportions have been decreasing in recent decades 23 . High proportions of ill-defined causes indicate limited access to health services or poor quality services, since they show that many individuals are dying without adequate diagnosis and treatment 24 .

In general, Brazilian children have shown an increase in the proportions of infant deaths from perinatal causes and congenital malformations, which already accounted for $68 \%$ of the causes of death in 1995-1997, along with a decrease in deaths from ARI and diarrhea 24 . In Guarani children under five years, practically all of the deaths were due to ARI and diarrhea. They accounted for nearly all of the defined causes of death in the 1-4-year bracket and for $90 \%$ of the defined causes under one year. Inequalities in proportional mortality due to ARI and diarrhea between indigenous peoples and the general population are large, especially for diarrhea in the South and Southeast among children 1-4 years of age and ARI in all regions of the country under one year of age.

Avoidable or reducible causes of death are those that are preventable through actions by health services 31 . More than half $(51.6 \%)$ of the Guarani deaths between zero and 75 years of age were due to preventable causes, and this proportion was somewhat lower between 5 and 75 years 
(41.7\%). Of these causes, $25 \%$ could be reduced by health promotion actions, prevention, and control and treatment of non-communicable diseases and $16.7 \%$ by immunization. In the group under five years, $77.8 \%$ of the deaths were potentially avoidable: $55.5 \%$ by adequate diagnosis and treatment, $16.6 \%$ by health promotion measures linked to health care, and $5.6 \%$ by prenatal and childbirth care.

Adequate treatment of pneumonia at the primary care level could reduce deaths from this cause by $70 \%$ in children under five years through actions such as active case search in the community, comprehensive and timely coverage of cases that require antibiotic therapy, referral of severe cases for hospital care according to protocol, and continuing training and supervision of health workers and integration among the levels of complexity in the health system, thus guaranteeing comprehensive health care 32,33. Therefore, the principal causes of Guarani mortality, especially under five years of age, would be potentially reducible by adequate diagnosis and treatment and health promotion actions 31 .

Vaccination is one of the main strategies for prevention and control of ARI and reduction of mortality from pneumonia $34,35,36,37$. The immunization schedule for indigenous peoples in Brazil is more extensive, differing from the basic schedule recommended by the Ministry of Health for the general population. At the time of this study, it included immunization against type B Haemophilus influenzae, Streptococcus pneumoniae (23-valent), and influenza virus, in addition to various other vaccines like BCG and triple bacterial (diphtheria, tetanus, and pertussis) and triple viral (measures, mumps, and rubella). Given the Guarani mortality pattern, one might question the coverage and effectiveness of vaccination in these indigenous communities, or even hypothesize that these deaths were due to infection by other agents like respiratory syncytial virus (RSV), which has high infant morbidity and mortality ( $<1$ year). Other hypotheses would include limited access and poor quality of care for prevalent cases of ARI, aggravated by the convergence of multiple social, cultural, and environmental health determinants facilitating the occurrence of respiratory diseases 20,21,34.

The majority of deaths from ARI in the Guarani occurred in hospital. This may have been due to late access to the hospital or even the quality and effectiveness of the hospital care 32 . One current strategy to expand the reach and to reduce deaths from pneumonia, diarrhea, malaria, measles, and malnutrition in children under five years is Integrated Management of Childhood Illness (IMCI), which aims to promote improvements in the health system and strengthen disease prevention and health promotion activities 38 . Local adaptations of the IMCI manuals, with appropriate training and supervision of health workers, could lead to safer and more effective management of pneumonia at the community level, especially where referral to hospitals is hindered by geographic, financial, or cultural factors 32 . Inadequate infrastructure, limitations to essential inputs, and the crisis in the health workforce have been identified as the principal limiting issues in the impact of interventions targeting management of pneumonia at the community level 32, all of which are prevalent conditions in the SIASI in Brazil.

\section{Final remarks}

The comparisons made by this study reveal important inequalities, unfavorable to the Guarani, resulting from distinct demographic patterns and differences in the exposure of indigenous peoples and the general population in Brazil to socioeconomic, cultural, and environmental determinants, including guarantees of subsistence and health care access and quality.

The systematic recording to vital events by the study's surveillance system allowed confirming the relevance of mortality from ARI and the vulnerability of the under-five-year age group. The predominance of avoidable causes of death and post-neonatal deaths suggests precarious living conditions and insufficient organization of indigenous health services, thus highlighting the need for greater investments in primary care and health promotion for indigenous peoples in order to improve their quality of life. This emphasizes the need for systematic, qualified recording of demographic and health data in order to support the planning and evaluation of health care for indigenous peoples. Such actions should be based on a cross-sectorial approach and include effective participation by the indigenous peoples themselves. 


\section{Resumo}

Mundialmente, povos indígenas apresentam elevada carga de doença, expressa por profundas iniquidades em saúde na comparação com os não-indígenas. Neste estudo, descreve-se a mortalidade Guarani no Sul e Sudeste do Brasil, com foco nas iniquidades em saúde. A estrutura populacional Guarani expressa elevada natalidade, mortalidade precoce e baixa idade mediana e esperança de vida ao nascer. A taxa de mortalidade bruta (TM bruta $=5,0 / 1.000$ ) se assemelha à nacional, mas a $T M<5$ anos $(44,5 / 1.000)$ e a taxa de mortalidade infantil (29,6/1.000) são duas vezes maiores que as TM correspondentes nas regiões Sul e Sudeste. A proporção de óbitos infantis pós-neonatais foi de 83,3\%, 2,4 vezes maior que a população geral. As proporções de causas mal definidas $(15,8 \%)$ e de evitáveis pelos serviços de saúde $(51,6 \%)$ foram elevadas. As principais causas de morte foram as respiratórias $(40,6 \%)$ e as infecciosas e parasitárias (18,8\%), sugerindo precárias condições de vida e de organização dos serviços de saúde. Há necessidade de maiores investimentos na atenção primária e em intervenções sobre os determinantes sociais da saúde, a fim de reduzir as iniquidades reveladas.

Índios Sul-Americanos; Infecções Respiratórias; Mortalidade; Desigualdades em Saúde

\section{Contributors}

A. M. Cardoso conceived the study, coordinated the data collection, and conducted the data analysis and interpretation, revision of the intellectual content, writing of the article, final revision, and approval and submission of the final version for publication. C. E. A. Coimbra Jr. collaborated in the study conceptualization, data interpretation, writing, final revision, and approval of the final version for publication. C. T. G. Barreto collaborated in the coordination of the data collection, data analysis, and approval of the final version for publication. G. L Werneck contributed to the data analysis, final revision, and approval of the final version for publication. R. V. Santos collaborated in the data interpretation, writing, final revision, and approval of the final version for $\mathrm{pu}-$ blication.

\section{Acknowledgments}

The authors wish to thank the Regional Offices of the FUNASA in the South and Southeast of Brazil, particularly the teams of the Indigenous Health Advisory Divisions; the EMSI, especially the registered nurses and other nursing staff; and above all the Guarani, who supported and believed in the work as a potential contribution to their children's health. The study was funded by FAPERJ (E-26/102.818/2008) and CNPq (grants no. 402522/2008-7 and no. 40.9677/2006-0).

\section{References}

1. Gracey M, King M. Indigenous health part 1: determinants and disease patterns. Lancet 2009; 374: 65-75.

2. King M, Smith A, Gracey M. Indigenous health part 2: the underlying causes of health gap. Lancet 2009; 374:76-85.

3. Montenegro RA, Stephens C. Indigenous health in Latin America and the Caribbean. Lancet 2006; 367:1859-69.

4. Barreto ML, Carmo EH. Padrões de adoecimento e de morte da população brasileira: os renovados desafios para o Sistema Único de Saúde. Ciênc Saúde Coletiva 2007; 12 Suppl:1779-90.

5. Noronha JC, Pereira TR, Viacava F. As condições de saúde dos brasileiros: duas décadas de mudanças (1980-2000). In: Lima NT, Gerschman S, Edler FC, Suárez JM, organizadores. Saúde e democracia. História e perspectivas do SUS. Rio de Janeiro: Editora Fiocruz; 2005. p. 153-92.

6. Duarte CMR. Reflexos das políticas de saúde sobre as tendências da mortalidade infantil no Brasil: revisão da literatura sobre a última década. Cad Saúde Pública 2007; 23:1511-28.

7. Curioni C, Cunha CB, Veras RP, André C. The decline in mortality from circulatory diseases in Brazil. Rev Panam Salud Pública 2009; 25:9-15.

8. Aquino R, Oliveira NF, Barreto ML. Impact of the Family Health Program on infant mortality in Brazilian municipalities. Am J Public Health 2009; 99:87-93.

9. Volpe FM, Abrantes MM, Capanema FD, Chaves JG. The impact of changing health indicators on infant mortality rates in Brazil, 2000 and 2005. Rev Panam Salud Pública 2009; 26:478-84.

10. Barros FC, Matijasevich A, Requejo JH, Giugliani E, Maranhão AG, Monteiro CA, et al. Recent trends in maternal, newborn, and child health in Brazil: progress toward Millennium Development Goals 4 and 5. Am J Public Health 2010; 100:1877-89.

11. Cardoso AM, Santos RV, Coimbra Jr. CEA. Mortalidade infantil segundo raça/cor no Brasil: o que dizem os sistemas nacionais de informação? Cad Saúde Pública 2005; 21:1602-8.

12. Garnelo L, Macedo G, Brandão LC. Os povos indígenas e a construção das políticas de saúde no Brasil. Brasília: Organização Pan-Americana da Saúde; 2003.

13. Pagliaro H, Carvalho NS, Rodrigues D, Baruzzi RG. Demographic dynamics of the Suyá, a Jê people of the Xingu Indigenous Park, Central Brazil, 19702004. Cad Saúde Pública 2007; 23:1071-81.

14. Pagliaro H. A revolução demográfica dos povos indígenas: a experiência dos Kaiabi do Parque Indígena do Xingu, Mato Grosso. In: Pagliaro H, Azevedo MM, Santos RV, organizadores. Demografia dos povos indígenas no Brasil. Rio de Janeiro: Editora Fiocruz/Associação Brasileira de Estudos Populacionais; 2005. p. 79-102.

15. Pena JL, Heller L, Dias Jr. CS. A população Xakriabá, Minas Gerais: aspectos demográficos, políticos, sociais e econômicos. Rev Bras Estud Popul 2009; 26:51-9. 
16. Souza LG, Santos RV, Coimbra Jr. CEA. Estrutura etária, natalidade e mortalidade do povo indígena Xavante de Mato Grosso, Amazônia, Brasil. Ciênc Saúde Coletiva 2010; 15 Suppl 1:1465-73.

17. Instituto Brasileiro de Geografia e Estatística. Tendências demográficas: uma análise dos indígenas com base nos resultados da amostra dos censos demográficos 1991 e 2000. Rio de Janeiro: Instituto Brasileiro de Geografia e Estatística; 2005.

18. Sousa MC, Scatena JHG, Santos RV. O Sistema de Informação da Atenção à Saúde Indígena (SIASI): criação, estrutura e funcionamento. Cad Saúde Pública 2007; 23:853-61.

19. Santos RV, Cardoso AM, Garnelo L, Coimbra Jr. CEA, Chaves MBG. Saúde dos povos indígenas e políticas públicas no Brasil. In: Giovanella L, Escorel S, Lobato LVC, Noronha JC, Carvalho AI, organizadores. Políticas e sistema de saúde no Brasil. Rio de Janeiro: Editora Fiocruz; 2008. p. 1035-56.

20. Cardoso AM. Doença respiratória aguda em indígenas Guarani no Sul e Sudeste do Brasil $[\mathrm{PhD}$ Dissertation]. Rio de Janeiro: Escola Nacional de Saúde Pública Sergio Arouca, Fundação Oswaldo Cruz; 2010.

21. Cardoso AM, Coimbra Jr. CEA, Tavares FG. Morbidade hospitalar indígena Guarani no Sul e Sudeste do Brasil. Rev Bras Epidemiol 2010; 13:21-34.

22. Organização Mundial da Saúde. Classificação estatística internacional de doenças e problemas relacionados à saúde - 10a Revisão. São Paulo: Centro Colaborador da OMS para Classificação de Doenças em Português/Faculdade de Saúde Pública, Universidade de São Paulo; 1995.

23. Rede Interagencial de Informação para a Saúde. Indicadores básicos para a saúde no Brasil: conceitos e aplicações. 2a Ed. Brasília: Organização PanAmericana da Saúde; 2008.

24. Victora CG, Cesar JA. Saúde materno-infantil no Brasil. Padrões de morbimortaliade e possíveis intervenções. In: Rouquayrol MZ, Almeida-Filho N, organizadores. Epidemiologia \& saúde. 6a Ed. Rio de Janeiro: MEDSI; 2003. p. 415-67.

25. Ricardo B, Ricardo F. Povos indígenas no Brasil: 2001-2005. São Paulo: Instituto Socioambiental; 2006.

26. Campanário P. Estimativas de fecundidade e de mortalidade de populações de pequena escala através de um modelo demográfico. In: Pagliaro H, Azevedo MM, Santos RV, organizadores. Demografia dos povos indígenas no Brasil. Rio de Janeiro: Editora Fiocruz/Associação Brasileira de Estudos Populacionais; 2005. p. 103-17.

27. Comissão Nacional sobre Determinantes Sociais da Saúde. As causas sociais das iniqüidades em saúde no Brasil. Comissão Nacional sobre Determinantes Sociais da Saúde. Rio de Janeiro: Editora Fiocruz; 2008.
28. Pagliaro H, Azevedo MM, Santos RV. Demografia dos povos indígenas no Brasil: um panorama crítico. In: Pagliaro H, Azevedo MM, Santos RV, organizadores. Demografia dos povos indígenas no Brasil. Rio de Janeiro: Editora Fiocruz/Associação Brasileira de Estudos Populacionais; 2005. p. 103-17.

29. Alcaraz-López GM. A fecundidade entre os Guarani: um legado de Kunhankarai [Doctoral Dissertation]. Rio de Janeiro: Escola Nacional de Saúde Pública Sergio Arouca, Fundação Oswaldo Cruz; 2000.

30. Machado M, Pagliaro H, Baruzzi RG. Demographic profile of the Hupd'äh, a Maku people living in the Upper Rio Negro Region, State of Amazonas, Brazil (2000-2003). Rev Bras Estud Popul 2009; 26:37-50.

31. Malta CD, Sardinha LM, Moura L, Lansky S, Leal MC, Szwarwald CL, et al. Update of avoidable causes of deaths due to interventions at the Brazilian Health System. Epidemiol Serv Saúde 2010; 19:173-6.

32. Theodoratou E, Al-Jilaihawi S, Woodward F, Ferguson J, Jhass A, Balliet M, et al. The effect of case management on childhood pneumonia mortality in developing countries. Int J Epidemiol 2010; 39:i155-71.

33. Chowdhury EK, El Arifeen S, Rahman M, Hoque DE, Hossain MA, Begum K, et al. Care at firstlevel facilities for children with severe pneumonia in Bangladesh: a cohort study. Lancet 2008; 372:781-2.

34. Mulholland EK, Smith L, Carneiro I, Becherc H, Lehmannd D. Equity and child-survival strategies. Bull World Health Organ 2008; 86:399-407.

35. Scott JAG, Brooks WA, Peiris JSM, Holtzman D, Mulholland EK. Pneumonia research to reduce childhood mortality in the developing world. J Clin Invest 2008; 118:1291-300.

36. Andre FE, Booy R, Bock HL, Clemens J, Datta SK, John TJ, et al. Vaccination greatly reduces disease, disability, death and inequity worldwide. Bull World Health Organ 2008; 86:140-6.

37. Sanders JW, Fuhrer GS, Johnson MD, Riddle MS. The epidemiological transition: the current status of infectious diseases in the developed world versus the developing world. Sci Prog 2008; 91:1-38.

38. El Arifeen S, Hoque DMH, Akter T, Rahman M, Hoque ME, Begum K, et al. Effect of the integrated management of childhood illness strategy on childhood mortality and nutrition in rural area in Bangladesh: a cluster randomized trial. Lancet 2009; 374:393-403.

Submitted on $16 / \operatorname{Jan} / 2011$

Final version resubmitted on 14/Apr/2011

Approved on 26/Apr/2011 